\title{
Role of astrocytes and microglia in central nervous system inflammation
}

\author{
Veit Rothhammer ${ }^{1} \cdot$ Francisco J. Quintana $^{1}$
}

Received: 31 July 2015 / Accepted: 3 August 2015 / Published online: 13 August 2015

(C) Springer-Verlag Berlin Heidelberg 2015

The role of the adaptive immune system in autoimmune inflammatory diseases of the central nervous system (CNS) has been strongly investigated during the past decades. However, a growing body of evidence supports the importance of CNS resident glial populations in the initiation, propagation, and regulation of inflammation. To provide insight into this fascinating development, this issue of Seminars in Immunopathology focuses on the functions of glial cells in the CNS during development, homeostasis, and disease.

Starting from embryonic development, the blood brain barrier (BBB) and its functions in health and disease are governed by close interactions between endothelial cells, astrocytes, and microglia. Thus, in the first review, Prat and coworkers highlight the importance of glial cells for the formation and regulation of the BBB, which establishes the anatomic and functional basis for the immunoprivileged status of the CNS.

Brendecke and Prinz discuss the diversity of CNS resident, adjoining and infiltrating myeloid cells during inflammation. As "guardians of the CNS", microglia, the resident macrophage population in the CNS, as well as strategically located myeloid cell subsets in the meninges, the perivascular space and the choroid plexus provide immune surveillance and shape the inflammatory response within and at the borders of the CNS.

This article is a contribution to the Special Issue on : Role of Astrocytes and Microglia in CNS Inflammation - Guest Editor: Francisco Quintana

Francisco J. Quintana

fquintana@rics.bwh.harvard.edu

1 Ann Romney Center for Neurologic Diseases, Brigham and Women's Hospital, Harvard Medical School, 77 Avenue Louis Pasteur, Boston, MA 02115, USA
Gold and El-Khoury review the role of microglia and the inflammasome in Alzheimer's disease (AD) and outline immunologic aspects of this neurodegenerative disorder. Indeed, this work highlights the dichotomous role of microglia, which alternates between protective clearance of $\beta$-amyloid and detrimental neurotoxic effects evoked by the activation of the NLRP3 inflammasome by $\beta$-amyloid. These mechanisms provide a new aspect on the role of microglia during the pathogenesis of AD.

Shemer and Jung focus on the role of resident microglia and infiltrating monocytes in CNS autoimmune diseases and emphasize therapeutic options targeting functional differences between these populations: infiltrating monocytes are critical for the effector phase of autoimmune CNS inflammation and actively initiate demyelination; however, microglia activation is required for monocyte recruitment to the CNS and precedes immune cell infiltration. These observations support therapeutic interventions targeting either microglial activation or monocyte recruitment for the treatment of inflammatory diseases.

Rothhammer and Quintana discuss the role of astrocytes as an integral part of the blood brain barrier and a functionally heterogeneous CNS resident population. During autoimmune inflammatory processes, astrocytes are exposed to a plethora of cytokines and chemokines that modulate their response. In addition, astrocytes participate actively in the fine-tuning of the local inflammatory milieu. This review gives an overview of the most recent understanding about cytokine networks operational in astrocytes during autoimmune CNS inflammation and highlights potential targets for immunomodulatory therapies for multiple sclerosis (MS).

Finally, Healy, Michell-Robinson, and Antel give an overview of the effects of current disease-modifying therapies for MS on the regulation of human glia. Based on their ability to cross the $\mathrm{BBB}$, different drugs act on astrocytes, microglia, 
oligodendrocytes, and their progenitors directly or indirectly by modulation of the peripheral immune compartment. This review also highlights future strategies for progressive stages of the disease and the reversibility of existent disabilities.

In conclusion, this issue of Seminars in Immunopathology provides a multifaceted overview of the role of glial cell populations during physiologic and pathologic conditions in the CNS, linking basic neuroscience with therapeutic aspects relevant for neurologic diseases. We present this issue with the expectation that these reviews will shed light on the role of glial populations in health and disease and illuminate the development of efficacious therapies for neurologic diseases. 